\title{
Keap 1 represses nuclear activation of antioxidant responsive elements by Nrf2 through binding to the amino-terminal Neh2 domain
}

\author{
Ken Itoh, Nobunao Wakabayashi, Yasutake Katoh, Tetsuro Ishii, Kazuhiko Igarashi, \\ James Douglas Engel, ${ }^{1}$ and Masayuki Yamamoto ${ }^{2}$ \\ Center for Tsukuba Advanced Research Alliance and Institute of Basic Medical Sciences, University of Tsukuba, \\ Tsukuba 305-8577, Japan
}

Transcription factor Nrf2 is essential for the antioxidant responsive element (ARE)-mediated induction of phase II detoxifying and oxidative stress enzyme genes. Detailed analysis of differential Nrf2 activity displayed in transfected cell lines ultimately led to the identification of a new protein, which we named Keap1, that suppresses Nrf2 transcriptional activity by specific binding to its evolutionarily conserved amino-terminal regulatory domain. The closest homolog of Keap1 is a Drosophila actin-binding protein called Kelch, implying that Keap1 might be a Nrf2 cytoplasmic effector. We then showed that electrophilic agents antagonize Keap1 inhibition of Nrf2 activity in vivo, allowing Nrf2 to traverse from the cytoplasm to the nucleus and potentiate the ARE response. We postulate that Keap1 and Nrf2 constitute a crucial cellular sensor for oxidative stress, and together mediate a key step in the signaling pathway that leads to transcriptional activation by this novel Nrf2 nuclear shuttling mechanism. The activation of Nrf2 leads in turn to the induction of phase II enzyme and antioxidative stress genes in response to electrophiles and reactive oxygen species.

[Key Words: ARE; electrophile; Keap1; Nrf2; oxidative stress]

Received August 14, 1998; revised version accepted November 10, 1998.

Both electrophiles and reactive oxygen species (ROS) contribute to DNA damage and consequent development of malignancy, as well as to many other diseases (Miller 1970, Sims et al. 1974; Ames 1983). To counteract these insults, terrestrial organisms have developed elaborate defense mechanisms (Bannai 1984; Prestera et al. 1993a; Primiano et al. 1997), which include the coordinated induction of a battery of specific genes that encode phase II detoxifying enzymes and oxidative stressinducible proteins (Buetler et al. 1995; Hayes and Pulford 1995). An illustration of how these defense mechanisms might work has been provided in model studies of carcinogenesis. Development of cancer in animals fed with strong chemical carcinogens is inhibited by the administration of low, but tolerable, concentrations of electrophilic agents (Wattenberg 1978). This protective phenomenon is referred to as the electrophile counterattack response (Prestera et al. 1993a), and is thought to be mediated by the induction of genes encoding phase II en-

\footnotetext{
${ }^{1}$ On academic leave from the Department of Biochemistry, Molecular Biology and Cell Biology, Northwestern University, Evanston, Illinois 60208-3500 USA.

${ }^{2}$ Corresponding author.

E-MAIL masi@tara.tsukuba.ac.jp; FAX 81-298-53-7318.
}

zymes (Primiano et al. 1997) and antioxidative stress proteins. This coordinated response is principally regulated through cis elements, called antioxidant responsive elements (AREs; Rushmore et al. 1991) or electrophile responsive elements (EpREs; Friling et al. 1990), associated with these target genes.

The ARE consensus sequence shows striking similarity to a binding motif referred to as the Maf recognition element (MARE), also known as the erythroid transcription factor NF-E2 binding sequence (Kataoka et al. 1994; Motohashi et al. 1997). MAREs are specifically recognized by either homodimers of Maf family members or by heterodimeric proteins composed of CNC

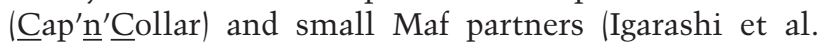
1994; Blank and Andrews 1997; Motohashi et al. 1997). The small Maf proteins lack canonical transcriptional activation domains, and hence the CNC factors provide the activation function for these heterodimers (Igarashi et al. 1994). The small Maf subunit however, is required for high affinity, sequence-specific DNA-binding activity of the CNC factors to the MARE element (Igarashi et al. 1994). The similarity between ARE and MARE sequences strongly implicated these families of proteins as candidate factors in mediating the ARE response. 
In accord with this expectation, we showed recently, by gene-targeted disruption in mice, that the CNC family member Nrf2 is a general regulator of the phase II enzyme genes (Itoh et al. 1997). More recently, we found that the induction of a repertoire of oxidative stress responsive genes is severely impaired in Nrf2-deficient macrophages (T. Ishii, K. Itoh, S. Takahashi, H. Sato, T. Yanagawa, Y. Katoh, S. Bannai, and M. Yamamoto, in prep.). On exposure to electrophilic agents, the DNAbinding activity of Nrf2 is markedly induced, whereas the Nrf2 steady-state mRNA level remains constant. These results suggested that signals from oxidative stress agents are transduced through an unidentified cellular receptor to the Nrf2 protein, which in turn mediates the electrophile counterattack response. Thus clarification of the mechanism(s) controlling Nrf2 activity appeared to be central to the elucidation of how terrestrial organisms sense destructive oxidative stress and subsequently mobilize an intrinsic cellular defense.

To explore the molecular mechanisms that activate Nrf2 and thereby transduce oxidative stress signals, we initially analyzed the structure and functional domains of the Nrf2 protein. Comparison of the human Nrf2 (hNrf2; Moi et al. 1994), chicken ECH (cNrf2; Itoh et al. 1995) and mouse Nrf2 (mNrf2; Chui et al. 1995) molecules showed that they were cross-species homologs; thus they are referred to simply as Nrf2 hereafter. In turn, this comparison, led to the experimental localization of a domain within the cNrf2 molecule that was capable of negatively regulating its activity in transfected cells. We then found that this domain associates with a novel cytoplasmic protein that directly negatively regulates the transactivation potential of Nrf2. We named this novel protein Keap1 (Kelch-like ECH-associated protein 1) because of its structural similarity to a Drosophila actin-binding protein called Kelch (Xue and Cooley 1993). We then showed that electrophilic agents functionally liberate Nrf2 transactivation activity from repression by Keap1, and concomitantly, that Nrf2 physically recompartmentalizes from the cytoplasm to the nucleus. These data suggest that together, Keap1 and
Nrf2 comprise the primary sensor for cellular response to oxidative stress.

\section{Results}

\section{Functional characterization of Nrf2}

CNC family transcription factors bind to MAREs as heterodimers with their obligatory partner molecules, the small Maf proteins (Motohashi et al. 1997). In comparing the human and chicken Nrf2 amino acid sequences, we found six highly conserved regions and named these homology domains Neh1 to Neh6 (Nrf2-ECH homology) (Fig. 1A). The first conserved domain, Neh1, contains the CNC homology region and basic-leucine zipper domain. The amino and carboxyl termini of the proteins are also highly conserved, and are referred to as Neh2 and Neh3, respectively. Additionally, there are two conserved acidic domains (Neh4 and Neh5) as well as a serine-rich conserved region (Neh6).

On closer inspection, Neh2 appeared to be a composite domain that is structurally divisible into two subregions (Fig. 1B). The amino-terminal region of Neh2, consisting of 32 amino acids, is rich in hydrophobic residues and shows marked conservation with the amino-terminal region of both Nrf1 and the C. elegans Skn-1 transcription factors (Bowerman et al. 1993). In contrast, the carboxyterminal half of Neh2, corresponding to amino acid residues $33-73$, is hydrophilic, and is not conserved among the CNC family members. The conservation of the carboxy-terminal half of Neh2 between species suggested that it might be an important functional domain specific to Nrf2.

On transient transfection into quail fibroblast cells (QT6), Nrf2 showed very strong transactivation activity, consistent with our previous analyses (Itoh et al. 1995; Toki et al. 1997). Therefore, we attempted to localize the transcription activation domain(s) of Nrf2. Various segments of Nrf2 were fused to the DNA binding domain (DBD) of the yeast transcription factor, Gal4 (Gal4-DBD; $\mathrm{Ma}$ and Ptashne 1987) to generate five Gal4-Nrf2 chimeric proteins (Fig. 2A; Gal4-Nrf2\#1 through Nrf2\#5).

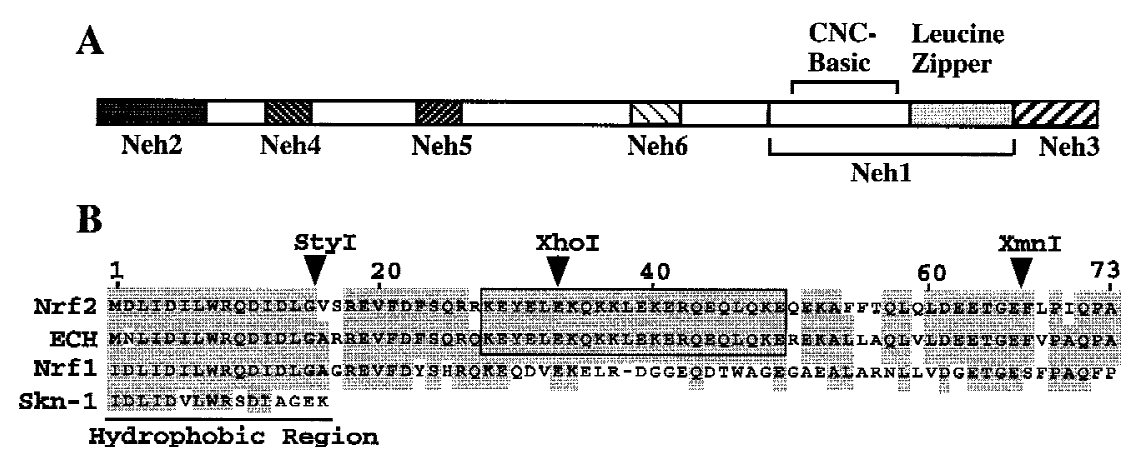

Figure 1. Schematic representation of the regions conserved between chicken and human Nrf2 proteins. (A) Six conserved domains, designated Neh1-Neh6, are found between human and chicken Nrf2. Neh1 corresponds to the CNC region and bZip structure. (B) Sequence homology in Neh2. Amino acid residues conserved between at least two proteins are shaded. The 33 amino-terminal residues, including the hydrophobic region, are conserved among Nrf1, hNrf2, and cNrf2 (ECH); the next 40 residues of Neh2 are rich in hydrophilic residues and specifically conserved between cross-species Nrf2 molecules. The strikingly homologous region, containing hydrophilic residues, is boxed. $(\boldsymbol{\nabla})$ Restriction enzyme sites. 
Figure 2. Transcriptional activation activity of Gal4 DBD-Nrf2 fusion proteins. $(A)$ Schematic presentation of the Gal4 DBDNrf2 fusion proteins. Gal4-Nrf2\#5 contains both Gal4 DBD and full-length chicken Nrf2 (ECH). (B) Transactivation activity of Nrf2 chimeric proteins. Trans-activation activity of each fusion protein was determined by cotransfection assay into the quail fibroblast cell line QT6, utilizing a LUC reporter plasmid, which is transcriptionally directed by five Gal4-binding sites.

Each column corresponds to the construct shown in A. LUC activity of the reporter plasmid in the absence of any effector plasmid was arbitrarily set at 100, and mean values of three independent experiments, each carried out in duplicate, are shown with the standard error of the mean (S.E.).

The activities of these fusion proteins were then examined in transient co-transfection assays with a minimal promoter/firefly luciferase $(L U C)$ reporter gene construct placed downstream of five Gal4-binding sites.

As shown in Figure 2B, Gal4-Nrf2 constructs 1 and 3 activated $L U C$ reporter gene transcription, but to a lesser extent than the full-length Gal4-Nrf2 fusion (Gal4Nrf2\#5). In contrast, Gal4-Nrf2\#2 and Gal4-Nrf2\#4 did not activate reporter gene expression. Equivalent expression of the chimeric proteins was routinely confirmed by immunoblot analysis with an anti-Gal4 antibody (data not shown). These results demonstrated that Nrf2 has two separable, independent, activation domains (Neh4 and Neh5) in fibroblasts, and that the Neh2 conserved homology domain is not an independent transactivation domain.

\section{Neh2 negatively regulates Nrf2 in erythroblasts}

To analyze the function of Neh2, we prepared a Nrf2 mutant from which the Neh2 domain was deleted (Fig. 3A). Wild-type (wild) and mutant (M1) Nrf2 cDNAs were independently cotransfected into QT6 quail fibroblasts or HD3 chicken proerythroblasts with the $L U C$ reporter construct pRBGP2, which contains three tandem MARE copies (Igarashi et al. 1994). Transfection of wild-type Nrf2 resulted in high levels of LUC activity in QT6 cells but only weak activation in HD3 cells (Fig. 3B,C). As anticipated from the previous transfection data localizing the Nrf2 transactivation domains (see above), deletion of Neh2 had very little effect on Nrf2 activity in transfected fibroblasts (Fig. 3B). Interestingly, the Neh2 deletion mutant was a much more potent transactivator than wild-type Nrf2 in erythroblasts (Fig. 3C). These results indicated that cNrf2 activity is normally repressed in HD3 proerythroblast cells and that the Neh2 domain is immediately involved in the observed cell type-specific negative regulation.

\section{Coexpression of a Neh2 decoy restores Nrf2 transactivation activity in transfected erythroblasts}

One hypothesis that might explain the deletion mutant analysis is that the Neh2 domain interacts with an as yet unidentified cellular protein that normally acts to repress Nrf2 activity. To experimentally test this hypothesis, we cotransfected Gal4-Nrf2\#2 plasmid, which encodes a Gal4-Neh2 fusion protein (see Fig. 2), together
Figure 3. The Neh2 domain is required for negative regulation of Nrf2 activity in HD3 erythroblasts. $(A)$ Schematic representation of the wild-type and Neh2 deletion mutant $(\mathrm{M} 1)$ of $\mathrm{cNrf2}$. $(B, C)$ Transfection of incremental amounts of the wild-type and mutant Nrf2 expression plasmids into QT6 cells or HD3 erythroblasts, respectively. (ロ) Wild; (O) M1. The pRBGP2 reporter, containing triplicated MARE binding sites from the chicken $\beta$-globin enhancer, was transfected simultaneously. LUC activity with the wild-type Nrf2 at an effector/reporter ratio of 20 was arbitrarily set at $100 \%$ in $B$, and that of the mutant at an effector/reporter ratio of 24 was set at $100 \%$ in $C$. Mean values of three independent experiments, each carried out in duplicate, are shown with the S.E.

\section{$\mathbf{A}$}

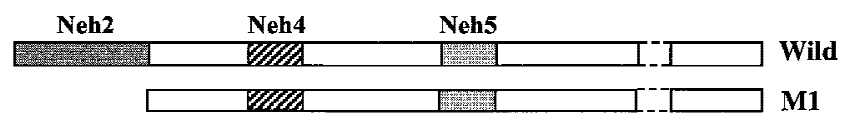

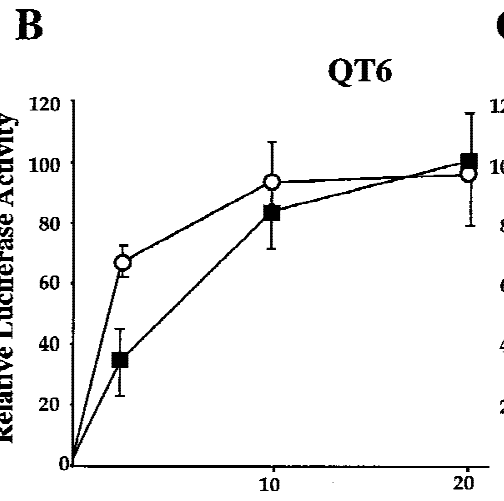

Effector/ Reporter Ratio
C

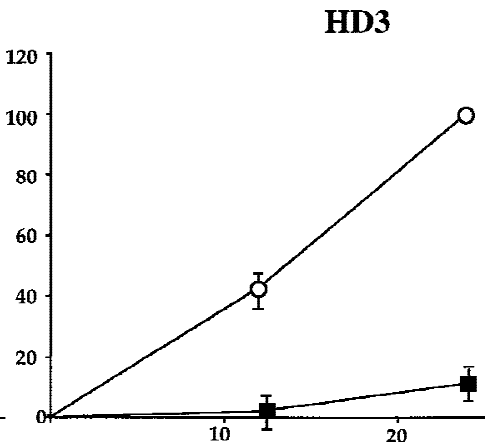

Effector/ Reporter Ratio 
with both the full-length Nrf2 expression plasmid and the $p R B G P 2$ reporter gene, into HD3 cells. As shown in Figure 4, LUC expression increased steadily when increasing amounts of Gal4-Neh2 plasmid were added to the transfections, whereas no similar titration occurred with a Gal4-DBD control plasmid, indicating that the expression of the Neh2 domain unmasked cryptic Nrf2 activity in HD3 cells. Importantly, cotransfection of Gal4-Neh2 did not influence the transactivation activity of the Nrf2 mutant protein that lacks Neh2 (the M1 mutant in Fig. 3; data not shown), indicating that the Gal4Neh2 fusion protein served as a decoy for competitive binding to a specific cellular activity. As an additional control, a chimeric protein consisting of Gal4 fused to the amino-terminal region of $\mathrm{Nrf1}$ (see Fig. 1B) failed to affect the transactivation activity of Nrf2 in HD3 erythroblasts (data not shown). These data thus implicate the presence of a cellular factor that serves as a negative regulator of Nrf2, which-on association with Nrf2 through the Neh2 domain-specifically inhibits Nrf2 activity in HD3 cells.

\section{Isolation of Keap1, a negative effector of Nrf2}

To identify the negative regulator of Nrf2, we used the Gal4-Nrf2\#2 protein, which contains the Gal4 DBD fused in-frame to Neh2 (see Fig. 2) as bait in the yeast two-hybrid system. We analyzed 80 of 300 recovered clones that activated reporter genes in conjunction with the bait plasmid from $2 \times 10^{7}$ yeast cells transformed with both the bait and a 17-dpc mouse embryo cDNA

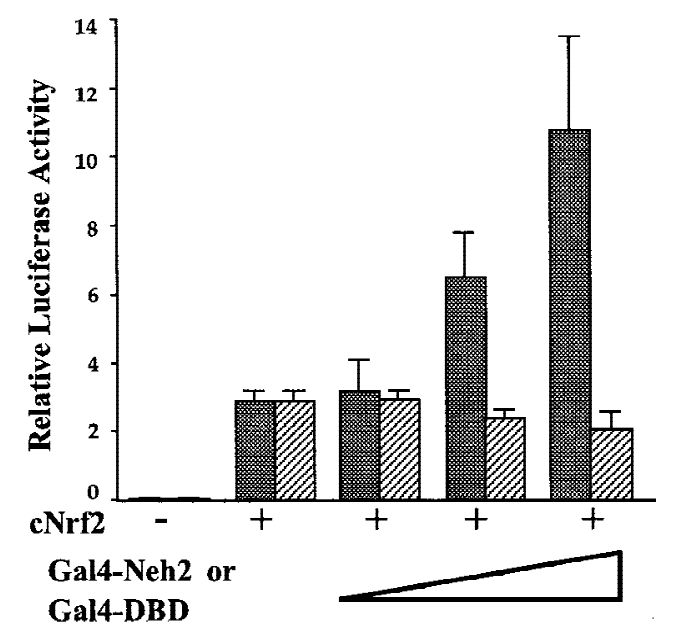

Figure 4. Overexpression of Gal4 DBD-Neh2 restores Nrf2dependent activation in HD3 cells. The full-length cNrf2 expression plasmid $(4 \mu \mathrm{g})$ was cotransfected into HD3 cells together with the pRBGP2 reporter $(0.5 \mu \mathrm{g})$ in the presence of increasing concentrations of expression plasmids encoding either Gal4 DBD-Neh2 or Gal4 DBD. LUC activity in the absence of effector plasmids was arbitrarily set at 0.1 . Mean values of three independent experiments, each carried out in duplicate, are shown with the S.E.. Note that the addition of Gal4 DBDNeh2 releases Nrf2 activity from repression in HD3 cells. (Stippled bars) Gal 4-Neh2; (hatched bars) Gal4-DBD.
A

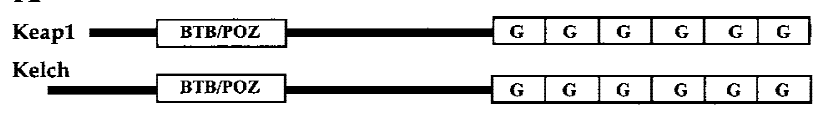

B

Keap1 MQPEPKLSGAPRSSQLPLWSKCPEGAGDAMMASHEGAEYTPSQDGNTTSYT KIAA0132 MQPDPRPSGAGACCRFLPLQSQGPEGAGDAWMASTECAEYYPSQHGNRTFSYT

LEOHTKQAFGMNELELSQQLCDVTLQVKYDDTPAAQFMAHKWLASSSPVFKAMTWGLREQGW LEUTTKQAFG MNELRLSQOLCDVLOVKYQDAPAAQFMAEKKULASSSPVFKAMFTNGLREQGW

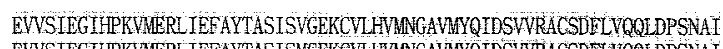
EWSTEGTHPKVERLIEFAYTASISMGEKCWLWMNGAVMQIDSWVACSDFLVQQDDPSAI

GIANFAEQIGCTELHQRAREYIYMH GEVAKOEEFHNSHCQLATLTSRDDNVREESEWHACI GIANEAEQIGCVELHQRAREYTYMHGEVAKQEEFHNLSCQLVTLT SRODENVRCESEVHACI

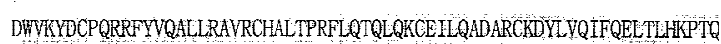

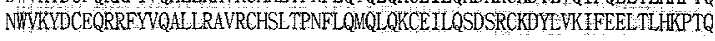

MPCRAPKVGRLYYTAGGYFROSLSYLEAMPSNGSWLRLADLOUPRSGLAGCWGGLEYVGGR WPCRAPNGRLIYTAGG YFRQSLSYLEAYNPSNGTLRLADLQWPRSGLAGCYGGLLYAVGGR

NASPDGNTDSSADDCYNPUTNQWSPCASMSVPRNRIGVGVIDGHYYVGGSHGCIHHSSVERYEP NOSPDGNTSSALDCYNPMTNQWSPCAPMSYPRNRIGVUDCHYAVGGSHGCHHNSVERYE

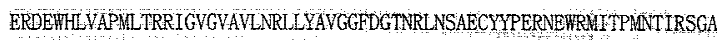



GVOLHNCIYAAGGYOCDQLNSVERYDVETETWTFVAPMRHHRSALGITHOGKIYVLGYDGH GVULHNCIYAAGCYDGQDQLASVERYVVTETWTVYPMKHRRSALGITVHQGR IYYLGGYDGY

TFLDSUECXDPDSDTWSEVTRMTSGRSGVGAVTMEPCRKQIDQQNCTC

TFLDSUECYDPDTOTWSEVTRMTSGRSGVGVAVMEPCRKQIDQQNCTC

Figure 5. Two-hybrid screening of Keap1. (A) Structural homology of Keap1 with the Drosophila Kelch protein, which contains BTB and DGR domains. Boxed Gs indicate the DGR domain. $(B)$ Keap1 shows high similarity to the human clone KIAA0132 (Nagase et al. 1995). Amino acid identities conserved between the two proteins are shaded.

prey library. The majority of these 80 clones encoded a single protein. Inspection of the conceptually translated primary amino acid sequence of the newly cloned cDNA revealed the presence of two canonical protein interaction motifs: a BTB domain (Bardwell and Treisman 1994) and a double glycine repeat (DGR) module (Albagli et al. 1995 ) in the middle and at the carboxy-terminal end of the protein, respectively (Fig. 5A). Database searches also revealed that this unusual combination of motifs is characteristic of the Drosophila cytoskeleton binding protein, Kelch (Xue and Cooley 1993); hence we named the new protein Keap1. The murine Keap1 protein also shows strong identity to a protein encoded by the human cDNA clone KIAA0132, which was isolated in a genome analysis project (Nagase et al. 1995). Because Keap1 shares $\sim 94 \%$ amino acid sequence identity with KIAA0132 (Fig. 5B), we concluded that the two are probably cross-species homologs.

To provide insight into the roles of Keapl in vivo, we first examined its expression profile in various mouse cell lines. As described above, DNA transfection analysis revealed only low Nrf2 transactivating activity in HD3 erythroblasts, suggesting that Keap 1 may be expressed more abundantly in hematopoietic cells than in fibroblasts. In confirmation of this expectation, RNA blot analysis showed that Keapl was highly expressed in he- 
matopoietic cell lines, but was less abundant in neuronal or fibroblastic cell lines (data not shown). Although this is consistent with the observed weaker transactivation potential of Nrf2 in hematopoietic cell lines, it should also be noted that Keap1 is expressed widely in various cell lines and tissues (data not shown), suggesting a ubiquitous, fundamental role for this protein.

To further define the region within Neh2 that interacts with Keap1, we next tested various fusion constructs of Neh2 (from cNrf2)-Gal4 DBD and Gal4 AD (activation domain)-Keap1 (Fig. 6A) in two-hybrid interaction assays. In these assays, if the bait constructs (B1-B4) interact with the AD constructs (L1-L3), yeast cells will acquire resistance to histidine-deficiency and colonize histidine deficient plates. Construct B1 contains full-length Neh2, whereas B4 lacks the hydrophilic

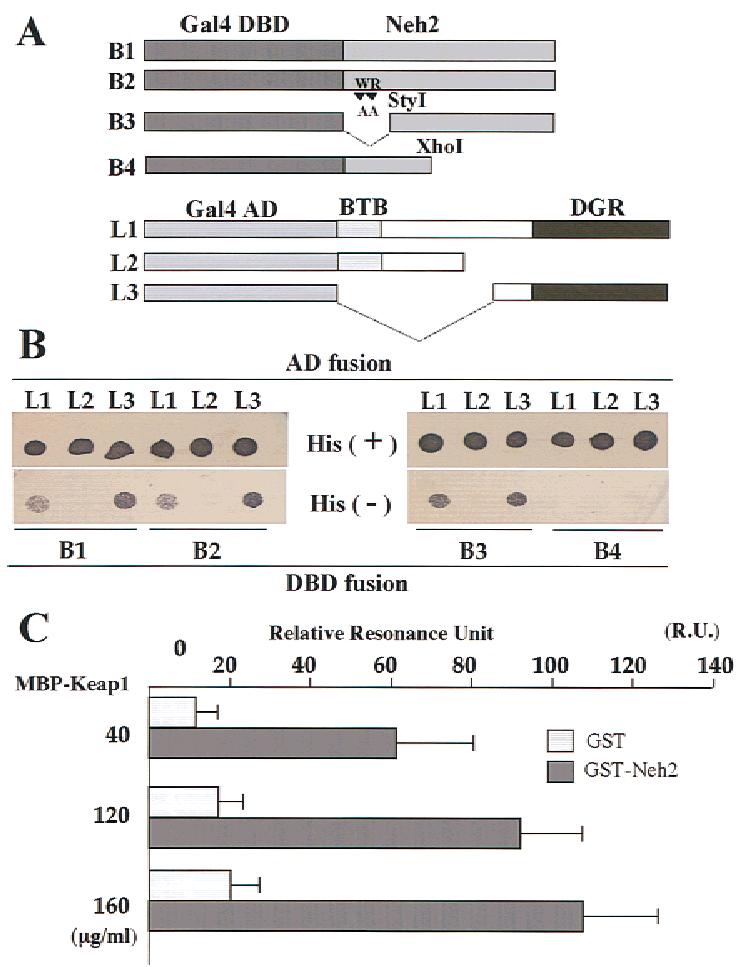

Figure 6. Interaction between the Neh2 domain of Nrf2 and the DGR domain of Keap1. (A) Schematic representations of the wild-type and mutant Gal4 DBD-Neh2 fusion proteins (B1 to $\mathrm{B} 4)$ and the Gal4 AD-Keap1 fusion proteins (L1-L3). Construct B1 contains full length Neh2. B4 is the same construct as the Neh2 deletion mutant of Nrf2 in Fig. 3. B2 contains two amino acid substitutions in Neh2, whereas B3 lacks the amino-terminal 15 residues. L2 and L3 lack the DGR or BTB domain of Keap1, respectively. (B) Expression plasmids for various Gal4 $\mathrm{DBD}$ and Gal4 $\mathrm{AD}$ fusion proteins were introduced into the reporter yeast strain in the given combinations. The resulting transformants were tested for the $\mathrm{His}^{+}$phenotype by spotting onto $\mathrm{His}^{-}$or His ${ }^{+}$plates. (C) Results of the BIAcore interaction assay. Ligand (GST-Neh2, amino acids 1-73, shaded bars) or GST alone (open bars), and analyte (MBP-Keap1-DGR, amino acids 308-624) binding was tested at three different concentrations. The test and the control values, each carried out in triplicate, are shown with the S.E. region (amino acids 33-73) of Neh2. B2 and B3 are both mutated in the amino-terminal 15 amino acid residues of Neh2; B2 contains 2 amino acid substitutions, whereas all 15 residues are deleted in mutant B3. Construct L1 contains both the BTB and DGR domains of Keap1 (Fig. $5 \mathrm{~A})$, whereas L2 and L3 lack either the DGR or the BTB domains, respectively. Baits B1, B2, and B3, but not B4, were found to interact with the L1 fusion protein (Fig. $6 \mathrm{~B})$, indicating that the hydrophilic region within the carboxy-terminal portion, but not the amino terminus, of Neh2 is necessary for association with Keap1. On the other hand, bait proteins B1-B3 were found to interact with the L3 protein, but not with the L2 protein, demonstrating that the DGR (glycine repeat) moiety of Keap1 is required for its interaction with Neh2. Thus, the integrity of the carboxyl terminus of Neh2 and the DGR motifs are essential for Keap1-Nrf2 association.

To examine the binding parameters governing Neh2Keap1 interaction in vitro, we utilized the BIAcore interaction assay (Pharmacia). Bacterially expressed GSTNeh2 and MBP-DGR fusion proteins were purified by affinity column chromatography. GST-Neh2 was fixed to a sensor chip coated with anti-GST antibody. MBPKeap1 was found to specifically interact with GST-Neh2 in a dose-dependent manner (Fig. 6C) but not with GST alone. The dissociation equilibrium constant $\left(K_{d}\right)$ for this interaction, calculated on the basis of the direct forward and reverse rate measurements (not shown), was determined to be $5.8 \times 10^{-7}$ (the average of three independent trials), thus indicating specific binding between the subdomains of these two proteins. In summary, these data demonstrate that the DGR domain of Keap1 and the Neh2 domain of Nrf2 physically associate both in vivo and in vitro.

\section{The Neh2 moiety is required for repression of Nrf2 activity by Keap1}

The results accumulated thus far suggested that forced expression of Keapl should repress the transactivation activity of Nrf2 in fibroblasts, just as it does in (normally) greater abundance in HD3 cells. To test this hypothesis, we transiently cotransfected the pRBGP2 reporter with either wild-type or mutant Nrf2 in the presence of increasing concentrations of Keap1 expression plasmid. As shown in Figure 7, A and B, Keap1 was found to repress the transactivation activity of both chicken and murine Nrf2 in a dose-dependent manner. In contrast, when Keap1 was cotransfected with Nrf2 deletion mutants lacking either the entire Neh2 (Fig. 3A; M1, amino acids $1-73$ ) or only the hydrophilic region (M2, amino acids 33-73), the transactivation potential of either Nrf2 mutant protein was not affected. These data unambiguously demonstrate that the repression of Nrf2 by Keap 1 is strictly dependent on the presence and integrity of the Neh2 moiety.

\section{Electrophilic agents abrogate Keap1 repression of Nrf2 activity}

We recently found that Nrf2 is necessary for the induc- 
Figure 7. Keap1 repression of Nrf2 activity. (A) Increasing amounts of Keap1 expression plasmid were cotransfected along with a constant amount of the pRBGP2 reporter and the wild-type or Neh2 deletion mutant (M1) cNrf2 expression plasmids into (normally Nrf2 permissive) QT6 cells. (B) Increasing amounts of Keap1 expression plasmid were transfected into QT6 cells along with a constant concentration of the wild-type mNrf2 or a second cNrf2 mutant (missing only the conserved hydrophilic core; amino acids 33-73) expression plasmid and the pRBGP2 reporter gene. LUC activity in the absence of any effector plasmid was arbitrarily set at 100 , and the mean values of three independent experiments, each carried out in duplicate, are shown with the S.E.
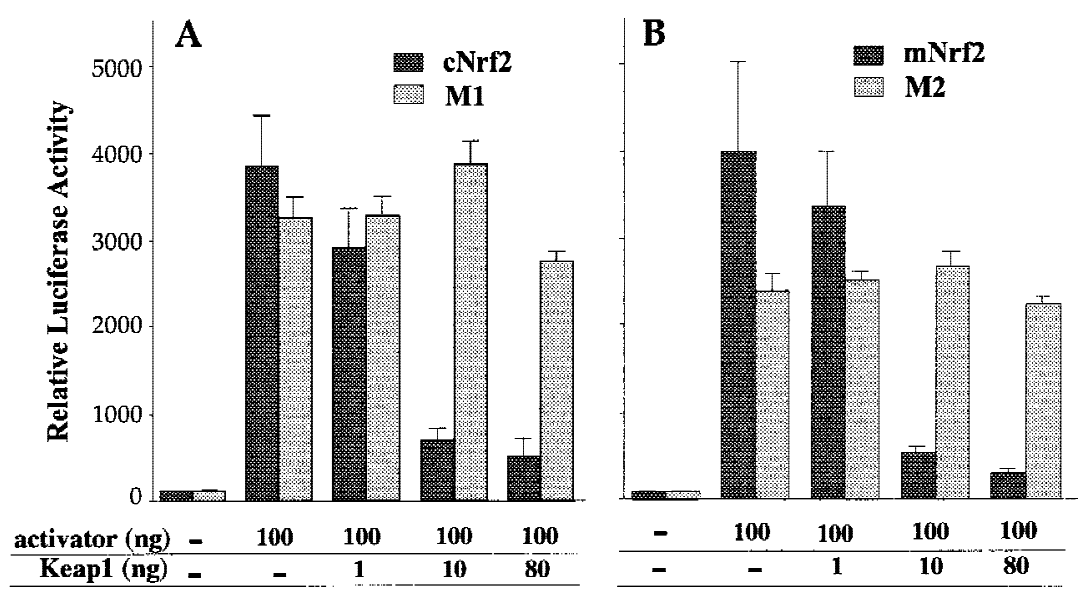

tion of a battery of oxidative stress-inducible genes ( $\mathrm{T}$. Ishii, K. Itoh, S. Takahashi, H. Sato, T. Yanagawa, Y. Katoh, S. Bannai, and M. Yamamoto, in prep.). For example, heme oxygenase 1 gene induction with the electrophilic agent diethylmaleate (DEM) is regulated by an Nrf2 small Maf heterodimer present in peritoneal macrophages. The data in the present study lend support to the hypothesis that an alliance between Keap1 and Nrf2 might constitute a sensor system for oxidative stress. This sensor may then mediate the signaling pathway that leads to the activation of Nrf2 and consequent transcriptional induction of the genes encoding phase II detoxifying enzymes and anti-oxidative stress proteins. To test this hypothesis, we cultured QT6 fibroblasts that had been transfected with the Keap1 and Nrf2 expression plasmids as well as the pRBGP2 reporter plasmid in the presence of DEM. The prediction is that Nrf2 activity should be liberated from Keap1 repression. As shown in
Figure 8A, the addition of DEM to the culture medium resulted in restoration of Nrf2 activity, despite the presence of Keap1, in a DEM dose-dependent manner.

As a control, we also transfected the pRBGP2 reporter alone or in combination with the Nrf2 expression plasmid into QT6 fibroblasts in the absence of cotransfected Keap1 expression plasmid, and cultured the cells with DEM. Without introducing exogenous Nrf2 in the transfected fibroblasts, we did not detect strong activation of the $L U C$ reporter gene, even in the presence of DEM (Fig. 8B). However, in the presence of cotransfected Nrf2, LUC reporter activity increased by $\sim 30 \%$ after addition of DEM (Fig. 8C). One plausible explanation for this observation is that electrophile treatment liberated Nrf2 activity from repression by endogenous Keap1. These results taken together support the notion that Keap1 and Nrf2 are the principal constituents of the pathway that transduce the electrophilic signal conferred by DEM
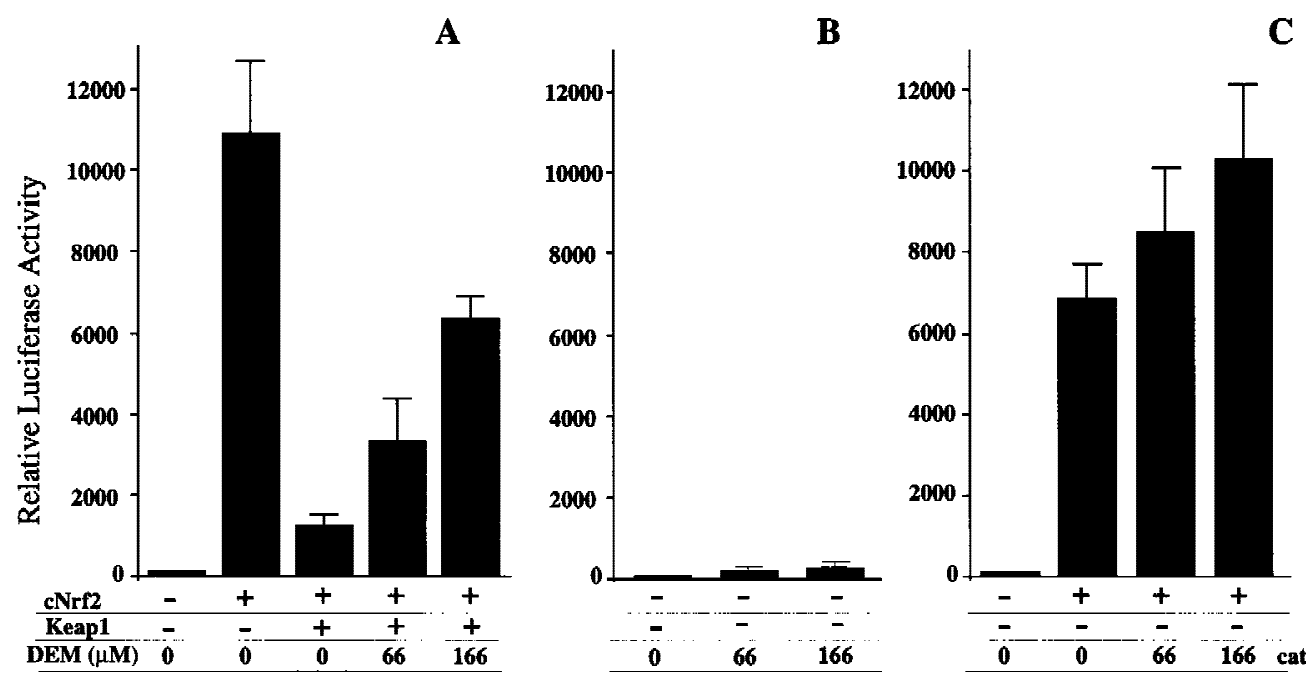

$\mathbf{C}$

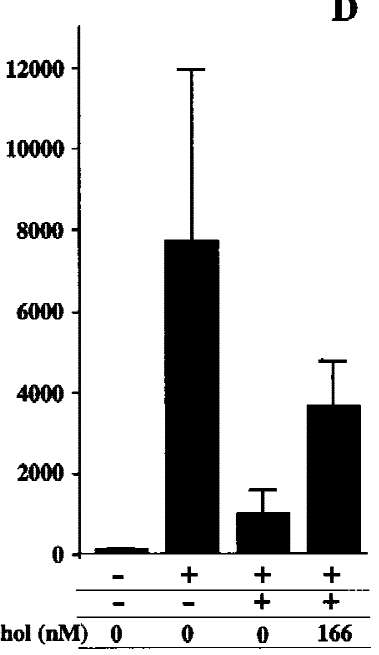

Figure 8. Electrophilic agents liberate Nrf2 from repression by Keap1. Both Nrf2 and Keap1 expression plasmids were transfected into QT6 fibroblasts. After $12 \mathrm{hr}$ of culture, the cells were washed with fresh medium, and then increasing amounts of DEM $(A)$ or catechol $(D)$ were added to the replacement medium. The QT6 cells were cultured for another $36 \mathrm{hr}$. LUC reporter activity of cells transfected with only pRBGP2 reporter $(B)$ or the reporter and the Nrf2 expression plasmid $(C)$, and treated with DEM, are also shown as controls. Results of three independent experiments, each of which were carried out in duplicate, are shown with the S.E. 
treatment to the inducible expression of phase II detoxifying enzyme genes and antioxidative stress protein genes.

We also tested another electrophilic agent, catechol, using the same assay, and concluded that catechol had the same effect as DEM within a very low concentration range (Fig. 8D). Thus the release of Nrf2 activity from Keap1-mediated repression is not restricted to DEM, but is a common response mechanism to electrophilic agents.

\section{Keap1 retains Neh2 reporter fusion proteins in the cytoplasm}

Because the Drosophila Kelch protein is a cytoskeleton binding protein (Xue and Cooley 1993), we thought that Keap 1 might function by retaining Nrf2 in the cytoplasm through interaction with the cytoskeleton. To examine the subcellular localization of Keap1, both Keap1-green fluorescent protein (GFP) and Flag-tagged Keap1 fusion constructs were prepared and transiently expressed in fibroblasts. The Keap1-GFP fusion protein was found to localize in the cytoplasm of transfected QT6 cells (Fig. 9B; A shows the expression profile of wild-type GFP) and NIH-3T3 cells (data not shown). The Flag-Keap1 fusion protein was also found to localize in the cytoplasm (data not shown). We examined the functional integrity of these fusion proteins by cotransfection of chicken or mouse Nrf2 expression plasmids and Keap1 plus the RBGP2 reporter plasmid. Both of the Keap1 chimeric proteins were competent in repressing the transactivation activity of Nrf2 (data not shown), thus indicating that the functional integrity of Keap1 was preserved in both fusion proteins.

Then we examined possible changes in the subcellular distribution of Neh2-GFP, which contains the negative regulatory domain of Nrf2 fused to GFP. Neh2-GFP was

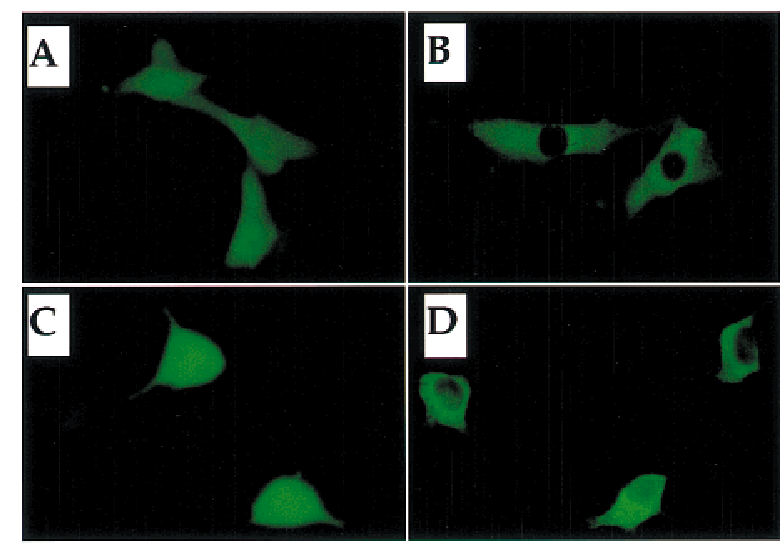

Figure 9. Subcellular localization of Keap1 and Neh2. $(A, B)$ Subcellular localization of Keap1-GFP. Expression plasmids for GFP alone $(A)$ or the Keap1-GFP fusion protein $(B)$ were transfected into QT6 cells. $(C, D)$ Subcellular localization of Neh2GFP. The expression plasmid for Neh2 (mNrf2)-GFP fusion protein was transfected into $293 \mathrm{~T}$ cells either alone $(C)$ or in combination with a plasmid expressing Keap1 $(D)$.

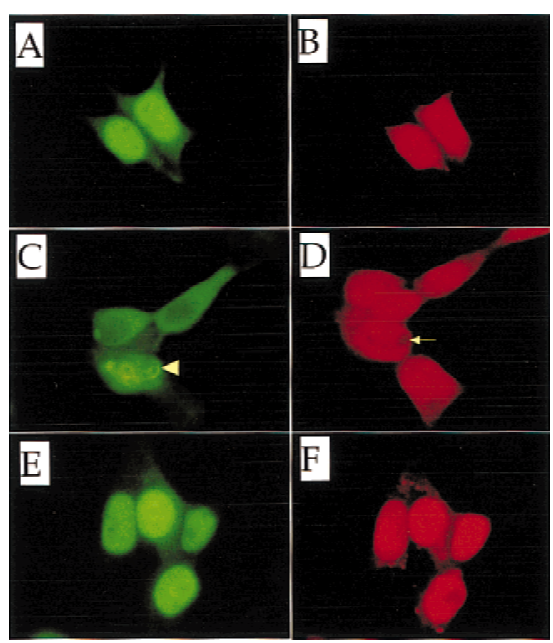

Figure 10. Subcellular localization of Nrf2. Nrf2 alone $(A, B)$ or both Nrf2 and Keap1 $(C, D)$ were force expressed in 293T cells. Nrf2 and Keap1 were also force-expressed in the presence of 100 $\mu \mathrm{M} \operatorname{DEM}(E, F) .(A, C, E)$ Localization of Nrf2 was detected using an anti-human Nrf2 antibody; $(B, D, F)$ the same fields stained with propidium iodide. The arrowhead and arrow in $C$ and $D$, respectively, show the characteristic perinuclear ring structure described in the text.

transfected with or without Keap1 expression plasmid into $293 \mathrm{~T}$ cells. Although cells transfected with Neh2GFP alone displayed relatively uniform fluorescence throughout the cells (including some staining in the nucleus; Fig. 9C), almost exclusively cytoplasmic fluorescence was observed when Neh2-GFP was cotransfected with Keap1 (Fig. 9D). These results are not at odds with the contention that Keap1 is a murine cytoskeleton binding protein, but more importantly, the data show that Keap1 acts to localize Nrf2, via Neh2, primarily in the cytoplasm.

\section{Electrophilic agents release the Nrf2 protein from the cytoplasm to the nucleus}

Given the preceding cellular localization observations, we wished to directly examine the cellular consequences of DEM treatment, whereupon Nrf2 activity is released from Keap1 repression, using an anti-Nrf2 antibody. When Nrf2 expression plasmid alone was transfected into $293 \mathrm{~T}$ cells, anti-Nrf2 immunoreactivity was detected predominately in the nucleus [Fig. 10A; B shows nuclei of the same cells stained with propidium iodide (PI)]. When Keap1 expression plasmid was cotransfected with the Nrf2 expression plasmid, the cellular localization of Nrf2 was principally in the cytoplasm (Fig. 10C,D). Entirely consistent with the Neh2-GFP observations described above, this result shows that Keap1 retains Nrf2 in the cytoplasm.

Finally, a potentially interesting, but currently not fully understood, observation was that some cells cotransfected with both Nrf2 and Keap1 expression plasmids displayed perinuclear ring structures (Fig. 10C; see 
arrowhead). Furthermore, these structures can also be visualized, and are colocalized, with PI staining (Fig. 10D; see arrow). Although the nature of these structures remains to be elucidated, cells displaying these ring structures have been observed reproducibly in this cotransfection analysis. The frequency of ring structure formation also diminished in the presence of DEM (below).

When DEM was added to the culture medium, Nrf2 was detected again in the nucleus even in the presence of cotransfected Keap1 (Fig. 10E,F). Thus, DEM enables Nrf2 to translocate from the cytoplasm to the nucleus. This observation extends, and is in very good agreement with, the results of the cotransfection/transactivation assay. These results argue that the Keap1-Nrf2 complex constitutes a cytoplasmic sensor system for oxidative stress.

\section{Discussion}

The ARE cis element has been shown to mediate responses to a variety of electrophilic agents (Prestera et al. 1993b), originally suggesting the existence of a common sensor system for induction by electrophiles. We demonstrated previously that nrf2 knockout mice are defective in the ARE response (Itoh et al. 1997). To begin to elucidate how a set of genes might be coordinately activated in response to oxidative stress signals, we examined the biochemical basis for Nrf2 activity in the present study. The DNA-binding activity of Nrf2 was enhanced with exposure of macrophages to electrophiles without any change in Nrf2 mRNA levels, demonstrating that regulation of Nrf2 activity was modulated posttranscriptionally. We also found that Nrf2 displayed quite different transactivation potential in different transfected cell lines and that Nrf2 repression was localized to a single domain that was common among murine, human, and chicken homologs. Localization of this specific, conserved domain in Nrf2 led in turn to the cloning and identification of the new regulatory partner Keap1. Keap1 was found to mediate Nrf2 repression by sequence-specific binding to this amino-terminal Neh2 domain of Nrf2. On the basis of these and other experimental observations, we concluded that Keap1 and Nrf2 together constitute the central cellular sensor for oxidative stress that mediate oxidative stress signals to the induction of phase II detoxifying enzyme genes and antioxidative stress protein genes.

Keapl has two discrete structural domains. One is DGR moiety (also known as the Kelch motif), whereas the other is the BTB protein interaction domain. Two other factors, ENC1 (Hernandez et al. 1997) and vaccinia A55 (Goebel et al. 1990), which contain similar structures, have also been reported. The discovery of the Neh2-DGR interaction and the cytoplasmic localization of Keap1 strongly suggest that Keap1 forms a multipartite complex, bridging Nrf2 and the actin cytoskeleton. Both Kelch and ENC proteins have been shown to contact the actin cytoskeleton through the DGR domain (Xue and Cooley 1993; Hernandez et al. 1997). In the absence of oxidative stress signals, Nrf2 is sequestered in the cytoplasm because of its association with Keap1. In addition, because the nuclear localization signal (NLS) does not lie within the Neh2 domain (K. Itoh and $M$. Yamamoto, unpubl.), we presume that NLS activity may also be masked in the Nrf2-Keap1 complex.

On exposure to oxidative stress or electrophiles, Nrf2 is released from repression mediated by Keap1 and translocates to the nucleus. This activating process is best illustrated in the transactivation and immunocytochemistry experiments examining Nrf2 subcellular localization in the presence or absence of Keap1 and DEM (or catechol). One can imagine that Keap1 might function in two very different ways to regulate Nrf2 transcriptional activity. One possible scenario is that an oxidative stress signal, acting either directly or indirectly, could release Keap1 from the cytoskeleton, thereby allowing it to traverse to the nucleus still associated with Nrf2. Alternatively, the signal could trigger the release of Nrf2 from Keap1, allowing Nrf2 to leave the cytoplasmic compartment and import into the nucleus, either alone or perhaps with another chaperone. Further studies will be necessary to elucidate the components and steps required for activation of Nrf2 by the electrophilic agents.

Galactose oxidase has DGR repeats that are similar to the Keap1 DGR motifs, and its crystal structure has been solved (Bork et al. 1994; Ito et al. 1994). On the basis of the structural information from galactose oxidase, we can project that the DGR motif of Keap1 contains a super-barrel structure consisting of six subcompartments, each of which generates a multi-interaction surface. Most interestingly, the DGR domain of galactose oxidase is involved in the electron transfer reaction (Bork et al. 1994; Ito et al. 1994). Because electrophilic agents activate Nrf2, the DGR domain of Keap1 may function in a similar fashion. This structural similarity thus suggests that the interaction interface between Keap1 and Nrf2 (and/or the actin cytoskeleton) may constitute the direct target of the oxidative stress signal, allowing Keap 1 to act as the primary sensor. Alternatively, it has also been suggested that the sensor system contains cysteine residues (Alam et al. 1995). Oxidation of reactive cysteine residues could cause critical conformational change in the protein, resulting in the release of any associated protein(s) (i.e., Nrf2). Importantly, Keap1 contains a considerable number of cysteine residues, and some of these appear to meet the criteria for reactive cysteines.

It is interesting to note that cytoskeleton-based cell signaling has been described in the pheromone and Hedgehog signaling pathways (Leeuw et al. 1995; Robbins et al. 1997; Sisson et al. 1997). The model we have proposed here shares some similarity with the Hedgehog signaling pathway, in which Costal2 anchors the transcription factor Cubitus interuptus in the cytoplasm through association with microtubules, in which yet unknown signals dissociate the complex, thus releasing the transcription factor to the nucleus. However, the mechanisms liberating Costal2 from microtubules still remain to be elucidated. 
In summary, the data presented here demonstrate the general regulatory scheme underlying the electrophile counterattack response. Electrophilic agents alter the interaction of Keap1 and Nrf2, thereby liberating Nrf2 activity from repression by Keap1, culminating in the induction of the phase II enzyme genes and antioxidative stress protein genes via AREs. Further elucidation of the tertiary structure of Keap1 and Nrf2 will illuminate the mechanism as to how Keap1 and Nrf2 respond and mediate the signals for oxidative stress.

\section{Materials and methods}

\section{Plasmid construction}

A series of Gal4-Nrf2 fusion protein plasmids were prepared for expression in animal cells (Gal4-Nrf2\#1 through Gal4-Nrf2\#4). The plasmids were generated by ligating the PCR-amplified portions of cNrf2 cDNA to pGBT9 (Clontech), in-frame, utilizing an EcoRI site and a SmaI site. Each construct was digested with HindIII, blunted by T4 DNA polymerase treatment, and subcloned into the BssHII site of pEF-BSSHII (Mizushima and Nagata 1994). Gal4-Nrf2\#5 was prepared by replacing the BamHIXbaI fragment of pCMV Gal4-Tax (the generous gift of Dr. W. Shoji, Tohoku University, Sendai, Japan) with an cNrf2 cDNA encoding the entire cNrf2 protein. The eukaryotic expression plasmids of wild-type and mutant cNrf2s were constructed by replacing the $5^{\prime}$ region of cNrf2 cDNA with PCR-amplified cNrf2 cDNA fragments encoding the deletion mutant proteins. The cNrf2 expression plasmids bearing internal deletions were generated by removing the respective portions of the cDNA by restriction enzyme digestion, as indicated in Figure 3A. Point mutations were created in the cNrf2 cDNA by a PCR-based mutagenesis method (Clontech).

\section{Transient transfection assays}

QT6 (Moscovici et al. 1997) and NIH-3T3 cells were maintained in DMEM supplemented with $10 \%$ fetal bovine serum (FBS) and seeded in 24-well dishes $24 \mathrm{hr}$ before transfection. The cells were transfected with the reporter and effector plasmids by use of calcium phosphate precipitation as described previously (Sambrook et al. 1989). The chicken proerythroblast cell line HD3 (Beug et al. 1982) was transfected with DEAE-dextran (Choi and Engel 1988). The LUC assay was performed by utilizing the Luciferase Assay System (Promega) following the supplier's protocol and assayed in a Biolumat Luminometer (Berthold). Transfection efficiencies were routinely normalized to the activity of a cotransfected $\beta$-galactosidase expression plasmid, pENL. Three independent experiments, each of which was carried out in duplicate, were performed normally, and the results were averaged and diagrammed with the S.E. indicated. DEM (Wako) or catechol (Sigma) was added to the culture medium immediately after the cells were washed at $12 \mathrm{hr}$ after transfection.

\section{Two-hybrid screening}

Saccharamyces cervisae two-hybrid screening was performed utilizing the Matchmaker Two-hybrid System (Clontech). A mouse 17 day-old embryonic Matchmaker cDNA library was transformed into HF7c yeast strain, along with Neh2 bait plasmid. Approximately $2 \times 10^{7}$ double transformants were tested for their ability to grow on $\mathrm{His}^{-}$medium. The HF7c cells were transformed with various combinations of plasmids that express $\mathrm{DBD}$ - or $\mathrm{AD}$-tagged molecules to examine the protein interactions within yeast cells. Yeast transformants were diluted in TE solution [10 $\mu \mathrm{M}$ Tris- $\mathrm{HCl}(\mathrm{pH} 8.0)$ and $1 \mathrm{~mm}$ EDTA] and spotted onto both $\mathrm{His}^{-}$and $\mathrm{His}^{+}$plates to test for the activation of the Gal4-dependent HIS3 reporter gene.

\section{Analysis of GFP fusion proteins}

The Keap1-GFP expression plasmid was generated by ligating the Keap1 cDNA to the amino terminus of GFP cDNA with the BgIII and HindIII sites in pEGFP (Clontech). The Neh2-GFP plasmid was constructed in a similar fashion. QT6 cells were transfected with the GFP or Keap1-GFP expression plasmids and incubated for $36 \mathrm{hr}$. The expression plasmid bearing the Neh2 (mNrf2)-GFP fusion protein was transfected into 293T cells either alone or in combination with that for Keap1. The cells were then cultured for $36 \mathrm{hr}$. After incubation, cells were washed once with PBS. Expression of GFP was examined with a Leica DMIRBE microscope equipped with an FITC filter for fluorescence detection.

\section{BIAcore assay}

Bacterial expression plasmids encoding Neh2 or Keap1 were constructed by ligating cNrf2 and Keap1 cDNAs to pGEX2T (Pharmacia) or pMalc-2 (New England Biolab), respectively. The BIAcore instrument (Pharmacia Biosensor AB, Uppsala, Sweden) was used as described (Natsume et al. 1994). Briefly, antiGST antibody was immobilized to the sensor chip surface with the GST capturing kit (Pharmacia). GST and GST-Neh2 (i.e., amino acids $1-73$ of cNrf2) were then bound separately to the immobilized GST antibody in PBS. The GST and GST-Neh2 interactions were tested with different concentrations of purified MBP-Keap1 (amino acids $308-624$ ) in PBS at $25^{\circ} \mathrm{C}$. The $K_{d}$ was calculated with BIAevaluation (v. 3.0) by the nonlinear fitting method following the manufacturer's recommendation.

\section{Immunocytochemistry}

293 T cells transiently expressing both Keap1 and Nrf2, or only Nrf2, were grown on slides in conditioned medium with or without $100 \mu \mathrm{M}$ DEM for $24 \mathrm{hr}$. The cells were then washed with PBS and fixed in $2 \%$ paraformaldehyde/lysine/periodate solution at room temperature for $10 \mathrm{~min}$ and then in $100 \%$ cold acetone for $10 \mathrm{~min}$ on ice. After washing with PBS, the cells were incubated with $2 \%$ goat serum for $1 \mathrm{hr}$ at $25^{\circ} \mathrm{C}$. The cells were first treated with rabbit anti-human Nrf2 antibody (1000fold dilution; Santa Cruz Biotechnology Inc.) for $16 \mathrm{hr}$ at $4^{\circ} \mathrm{C}$, and subsequently with FITC-conjugated goat anti-rabbit immunoglobulin antibody (Zymed) for $1 \mathrm{hr}$ at room temperature. After washing the cells with PBS, a tiny drop of fluorescent mounting medium (DAKO) containing $200 \mathrm{ng} / \mathrm{ml}$ PI was placed on the slides. The cells were then examined by fluorescence microscopy.

\section{Acknowledgments}

We thank Drs. Shiro Bannai, Kim Chew Lim, Roger Patient, Wataru Shoji, Kazuhiko Umesono, and Ruth Yu for help and discussion. This work was supported in part by Grants-in-Aid from the Ministry of Education, Science, Sports, and Culture, 
Core Research for Evolutional Science and Technology (CREST), the National Institutes of Health (CA 80088), and the Japanese Society for Promotion of Sciences (JSPS). K.I. is a research fellow of the JSPS and N.W. is a research fellow of CREST.

The publication costs of this article were defrayed in part by payment of page charges. This article must therefore be hereby marked 'advertisement' in accordance with 18 USC section 1734 solely to indicate this fact.

\section{Note added in proof}

The sequence of mouse Keap1 has been deposited in DDBJ/ GenBank under accession no. AB020063.

\section{References}

Alam, J., S. Camhi, and A.M.K. Choi. 1995. Identification of a second region upstream of the mouse heme oxygenase-1 gene that functions as a basal level and inducer-dependent transcriptional enhancer. J. Biol. Chem. 270: 11977-11984.

Albagli, O., P. Dhordain, C. Deweindt, G. Lecocq, and D. Leprince. 1995. The BTB/POZ domain: A new protein-protein interaction motif common to DNA- and actin-binding proteins. Cell Growth Differ. 6: 1193-1198.

Ames, B.N. 1983. Dietary carcinogens and anticarcinogens. Oxygen radicals and degenerative disease. Science 221: $1256-1264$

Bannai, S. 1984. Induction of cystine and glutamate transport activity in human fibroblasts by diethylmaleate and other electrophilic agents. J. Biol. Chem. 264: 2435-2440.

Bardwell, V.J. and R. Treisman. 1994. The POZ domain: A conserved protein-protein interaction motif. Genes \& Dev. 8: $1664-1677$.

Beug, H., G. Doederlien, C. Freudenstein, and T. Graf. 1982. Erythroblast cell lines transformed by a ts mutant of avian erythroblastosis virus: A model system to study erythroid differentiation in vitro. J. Cell. Physiol. (Suppl.) 1: 195-207.

Blank, V. and N.C. Andrews. 1997. The Maf transcription factors: Regulators of differentiation. Trends. Biochem. Sci. 22: 437-441.

Bork, P. and R.F. Doolittle. 1994. Drosophila Kelch motif is derived from a common enzyme fold. J. Mol. Biol. 236: $1277-$ 1282.

Bowerman, B., B.W. Draper, C.C. Mello, and J.R. Priess. 1993. The maternal gene skn-1 encodes a protein that is distributed unequally in early C. elegans embryos. Cell 74: 443452.

Buetler, T.M., E.P. Gallagher, C. Wang, D.L. Stahl, J.D. Hayes, and D.L. Eaton. 1995. Induction of phase I and phase II drug metabolizing enzyme mRNA, protein and activity by BHA, ethoxyquin and oltipraz. Toxicol. Appl. Pharmacol. 135: 4557.

Choi, O.R. and J.D. Engel. 1988. Developmental regulation of $\beta$-globin switching. Cell 55: 17-26.

Chui, D.H.K., W. Tang, and S.H. Orkin. 1995. cDNA cloning of murine Nrf2 gene, coding for a p45 NF-E2 related transcription factor. Biochem. Biophys. Res. Commun. 209: 40-46.

Friling, R.S., S. Bensimon, and V. Daniel. 1990. Xenobiotic-inducible expression of murine glutathione S-transferase Ya subunit gene is controlled by an electrophile-responsive element. Proc. Natl. Acad. Sci. 87: 6258-6262.

Goebel, S.J., G.P. Johnson, M.E. Perkus, S.W. Davis, J.P. Winslow, and E. Paoletti. 1990. The complete DNA sequence of
Vaccinia virus. Virology 179: 247-266.

Hayes, J.D. and J.D. Pulford. 1995. The glutathione S-transferase supergene family: Regulation of GST and the contribution of the isozymes to cancer chemoprevention and drug resistance. Crit. Rev. Biochem. Mol. Biol. 30: 445-600.

Hernandez, M-C., P.J. Andres-Barquin, S. Martinez, A. Bulfone, J.L.R. Rubenstein, and M.A. Israel. 1997. ENC-1: A novel mammalian Kelch-related gene specifically expressed in the nervous system encodes an actin-binding protein. J. Neuroscience 17: 3038-3051.

Igarashi, K., K. Kataoka, K. Itoh, N. Hayashi, M. Nishizawa, and M. Yamamoto. 1994. Regulation of transcription by dimerization of erythroid factor NF-E2 p45 with small Maf proteins. Nature 367: 568-572.

Ito, N., S.E.V. Phillips, K.D.S. Yadav, and P.F. Knowles. 1994. Crystal structure of a free radical enzyme, galactose oxidase. J. Mol. Biol. 238: 794-814.

Itoh, K., K. Igarashi, N. Hayashi, M. Nishizawa, and M. Yamamoto. 1995. Cloning and characterization of a novel erythroid cell-derived CNC family transcription factor heterodimerizing with the small maf family proteins. Mol. Cell. Biol. 15: 4184-4193.

Itoh, K., T. Chiba, S. Takahashi, T. Ishii, K. Igarashi, Y. Katoh, T. Oyake, N. Hayashi, K. Satoh, I. Hatayama, M. Yamamoto, and Y. Nabishima. 1997. An Nrf2/small Maf heterodimer mediates the induction of phase II detoxifying enzyme genes through antioxidant response elements. Biochem. Biophys. Res. Commun. 236: 313-322.

Kataoka, K., M. Noda, and M. Nishizawa. 1994. Maf nuclear oncoprotein recognizes sequences related to an AP-1 site and forms heterodimers with both Fos and Jun. Mol. Cell. Biol. 14: 700-712.

Leeuw, T., A. Fourest-Lieuvin, C. Wu, J. Chenevert, K. Clark, M. Whiteway, D.Y. Thomas, and E. Leberer. 1995. Pheromone response in yeast: Association of Bem $1 \mathrm{p}$ with proteins of the MAP kinase cascade and actin. Science 270: 12101213.

Ma, J. and M. Ptashne. 1987. A new class of yeast transcriptional activators. Cell 51: 113-119.

Miller, J.A. 1970. Carcinogenesis by chemicals: An overview. G.H.A. Clowes Memorial Lecture. Cancer Res. 39: 559-576.

Mizushima, S. and S. Nagata. 1994. pEF-BOS, a powerful mammalian expression vector. Nucleic Acids Res. 18: 5332.

Moi, P., K. Chan, I. Asunis, A. Cao, and Y.W. Kan. 1994. Isolation of NF-E2-related factor 2 (Nrf2), a NF-E2-like basic leucine zipper transcriptional activator that binds to the tandem NF-E2/AP1 repeat of the $\beta$-globin locus control region. Proc. Nat1. Acad. Sci. 91: 9926-9930.

Moscovici, C., M. Giovannella, M.H. Jimenez, M.M.C. Lai, M.J. Haman, and P.K. Vogt. 1997. Continuous tissue cell lines derived from chemically induced tumors of Japanese quail. Cell 11: 95-103.

Motohashi, H., J.A. Shavit, K. Igarashi, M. Yamamoto, and J.D. Engel. 1997. The world according to Maf. Nucleic Acids Res. 25: 2953-2959.

Nagase, T., N. Seki, A. Tanaka, K. Ishikawa, and N. Nomura 1995. Prediction of the coding sequences of unidentified human genes, IV. The coding sequences of 40 new genes (KIAA0121-KIAA0160) deduced by analysis of cDNA clones from human cell line KG-1. DNA Res. 2: 164-174.

Natsume, T., T. Koide, S. Yokota, K. Hirayoshi, and K. Nagata. 1994. Interaction between collagen-binding stress protein HSP47 and collagen. J. Biol. Chem. 269: 31224-31228.

Prestera, T., Y. Zhang, R.S. Spencer, A.C. Wilczak, and P. Talalay. 1993a. The electrophile counterattack response: Protection against neoplasia and toxicity. Adv. Enzyme Regul. 
Itoh et al.

33: 281-296.

Prestera, T., W.D. Holtzclaw, Y. Zhang, and P. Talalay. 1993b. Chemical and molecular regulation of enzymes that detoxify carcinogens. Proc. Natl. Acad. Sci. 90: 2965-2969.

Primiano, T., T.R. Sutter, and T.W. Kensler. 1997. Antioxidantinducible genes. Adv. Pharmacol. 38: 293-328.

Robbins, D.J., K.E. Nybakken, R. Kobayashi, J.C. Sisson, J.M. Bishop, and P.P. Therond. 1997. Hedgehog elicits signal transduction by means of a large complex containing the kinesin-related protein Costal2. Cell 90: 225-234.

Rushmore, T.H., M.R. Morton, and C.B. Pickett. 1991. The antioxidant responsive element. I. Biol. Chem. 266: 11632-11639.

Sambrook, J., E.F. Fritsch, and T. Maniatis. 1989. Molecular cloning. A laboratory manual. Cold Spring Harbor Laboratory Press, Cold Spring Harbor, NY.

Sims, P., P. L. Grover, A. Swaisland, K. Pal, and A. Hewer. 1974. Metabolic activation of benzo(a)pyrene proceeds by a diolepoxide. Nature 252: 326-329.

Sisson, J.C., K.S. Ho, K. Suyama, and M.P. Scott. 1997. Costal2, a novel kinesin-related protein in the Hedgehog signaling pathway. Cell 90: 235-245.

Toki, T., J. Itoh, J. Kitazawa, K. Arai, K. Hatakeyama, J. Akasaka, K. Igarashi, N. Nomura, M. Yokoyama, M. Yamamoto, and E. Ito. 1997. Human small Maf proteins form heterodimers with CNC family transcription factors and recognize the NF-E2 motif. Oncogene 14: 1902-1910.

Wattenberg, L.W. 1978. Inhibitors of chemical carcinogenesis. Adv. Cancer Res. 26: 197-226.

Xue, F. and L. Cooley. 1993. Kelch encodes a component of intracellular bridges in Drosophila egg chambers. Cell 72: 681-693. 


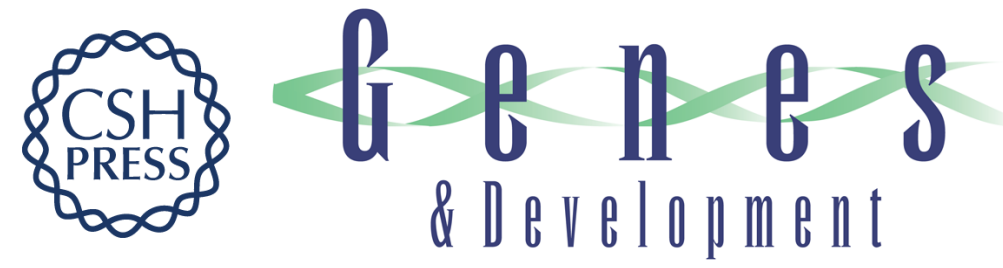

\section{Keap1 represses nuclear activation of antioxidant responsive elements by Nrf2 through binding to the amino-terminal Neh2 domain}

Ken Itoh, Nobunao Wakabayashi, Yasutake Katoh, et al.

Genes Dev. 1999, 13:

Access the most recent version at doi:10.1101/gad.13.1.76

$\begin{array}{ll}\text { References } & \begin{array}{l}\text { This article cites } 37 \text { articles, } 13 \text { of which can be accessed free at: } \\ \text { http://genesdev.cshlp.org/content/13/1/76.full.html\#ref-list-1 }\end{array}\end{array}$

License

Email Alerting Receive free email alerts when new articles cite this article - sign up in the box at the top Service right corner of the article or click here.

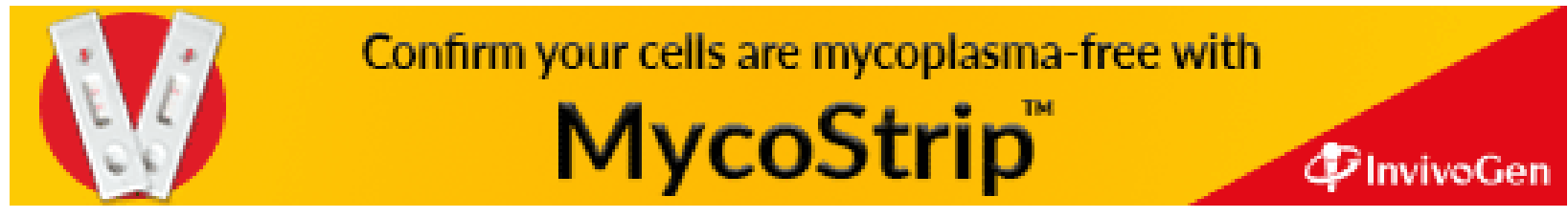

